\title{
A straightforward approach for bioorthogonal labeling of proteins and organelles in live mammalian cells, using a short peptide tag
}

\author{
Inbar Segal ${ }^{1,2}$, Dikla Nachmias ${ }^{1,2}$, Andres Konig ${ }^{1,2}$, Ariel Alon ${ }^{1,2}$, Eyal Arbely ${ }^{2,3}$ and Natalie Elia ${ }^{1,2^{*}}$ (D)
}

\begin{abstract}
Background: In the high-resolution microscopy era, genetic code expansion (GCE)-based bioorthogonal labeling offers an elegant way for direct labeling of proteins in live cells with fluorescent dyes. This labeling approach is currently not broadly used in live-cell applications, partly because it needs to be adjusted to the specific protein under study.

Results: We present a generic, 14-residue long, N-terminal tag for GCE-based labeling of proteins in live mammalian cells. Using this tag, we generated a library of GCE-based organelle markers, demonstrating the applicability of the tag for labeling a plethora of proteins and organelles. Finally, we show that the HA epitope, used as a backbone in our tag, may be substituted with other epitopes and, in some cases, can be completely removed, reducing the tag length to 5 residues.
\end{abstract}

Conclusions: The GCE-tag presented here offers a powerful, easy-to-implement tool for live-cell labeling of cellular proteins with small and bright probes.

\section{Background}

Tracking the dynamics of proteins and organelles in live cells is key to understanding their functions. For this, fluorescent protein (e.g., GFP) or self-labeling protein (e.g., Halo-Tag) tags are routinely attached to proteins in cells [1]. While these tags are vigorous and easy to implement, they are large and bulky (e.g., GFP, $27 \mathrm{kDa}$; Halotag, $33 \mathrm{kDa}$ ), such that their attachment could affect the dynamics and function of the protein under study. Using genetic code expansion (GCE) and bioorthogonal chemistry, it is now possible to attach fluorescent dyes (Fl-dyes) to specific protein residues, thereby allowing direct labeling of proteins in live cells with Fl-dyes [1-3]. Indeed, this approach has been applied, in recent years, for fluorescent labeling of extra- and intracellular proteins [4-10].

\footnotetext{
* Correspondence: elianat@post.bgu.ac.il

${ }^{1}$ Department of Life Sciences, Ben-Gurion University of the Negev, 84105 Beer Sheva, Israel

${ }^{2}$ National Institute for Biotechnology in the Negev (NIBN), Ben-Gurion University of the Negev, 84105 Beer Sheva, Israel

Full list of author information is available at the end of the article
}

In GCE-based labeling, a non-canonical amino acid (ncAA) carrying a functional group is incorporated into the sequence of a protein in response to an in-frame amber stop codon (TAG), via an orthogonal tRNA/tRNA-synthetase pair (reviewed in $[11,12]$ ). Labeling is then carried out by a rapid and specific bioorthogonal reaction between the functional group and the Fl-dye [2, 4, 8, 9, 13, 14]. Successful labeling hence relies on the exogenous expression of an orthogonal tRNA/tRNA-synthetase pair and a protein of interest (bearing a ncAA) at sufficient levels to allow effective labeling.

The ncAA (and consequently the Fl-dye) can, in theory, be incorporated anywhere in the protein sequence. In practice, however, finding a suitable labeling site can be laborious and time-consuming for several reasons. First, prior knowledge or functional assays are necessary to ensure that the insertion of the ncAA at a specific position does not affect protein structure and function $[4-7,10]$. Second, the efficiency of ncAA incorporation varies at different locations in the protein with no guidelines for the preferred sequence context having been reported $[3-7,15]$. Notably, 
low efficiency of ncAA incorporation does not only lead to ineffective labeling but also to the translation of a truncated version of the protein (resulting from the insertion of a premature stop codon), which can be toxic to cells $[5,6,16$, 17]. Third, the ncAA should be incorporated in a position that will allow the functional group to be accessible to the solvent to enable efficient bioorthogonal conjugation with the Fl-dye. All these requirements are protein specific, such that any attempt at labeling via this approach begins with a screen for suitable incorporation sites $[2-4,6,7]$. Consequently, despite its great potential, GCE-based labeling is presently not widely used in mammalian live-cell imaging studies [5].

To bypass the screening steps currently associated with GCE-based labeling and expand the use of the technique, we set out to design a minimal peptide tag that encodes the incorporation of a ncAA through GCE, for bioorthogonal labeling of proteins in live cells; we term this tag a GCE-tag (Fig. 1a). Using the HA epitope as a backbone, we optimized a 14-residue long N-terminal tag and demonstrated its applicability for live-cell labeling of proteins and organelle markers in mammalian cells. With this tag in hand, cellular proteins can now be labeled via GCE and bioorthogonal chemistry for live-cell imaging applications with no screening steps required.

\section{Results}

Proteins can potentially be tagged at their $\mathrm{N}$ - or Cterminal. For GCE-based labeling, we chose to design an $\mathrm{N}$-terminal tag in order to avoid protein truncations resulting from inefficient incorporation of the ncAA $[5,6,16$, 17]. On the basis of our previous work, potential tags were

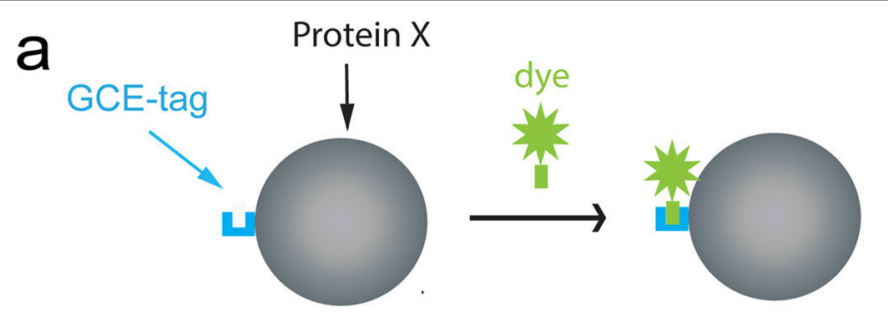

b GCE-tag Protein $X$ mRNA $\quad x x x$ UAG $x x x x x x x x x x x x$ xxx $x x x x x x x x x$

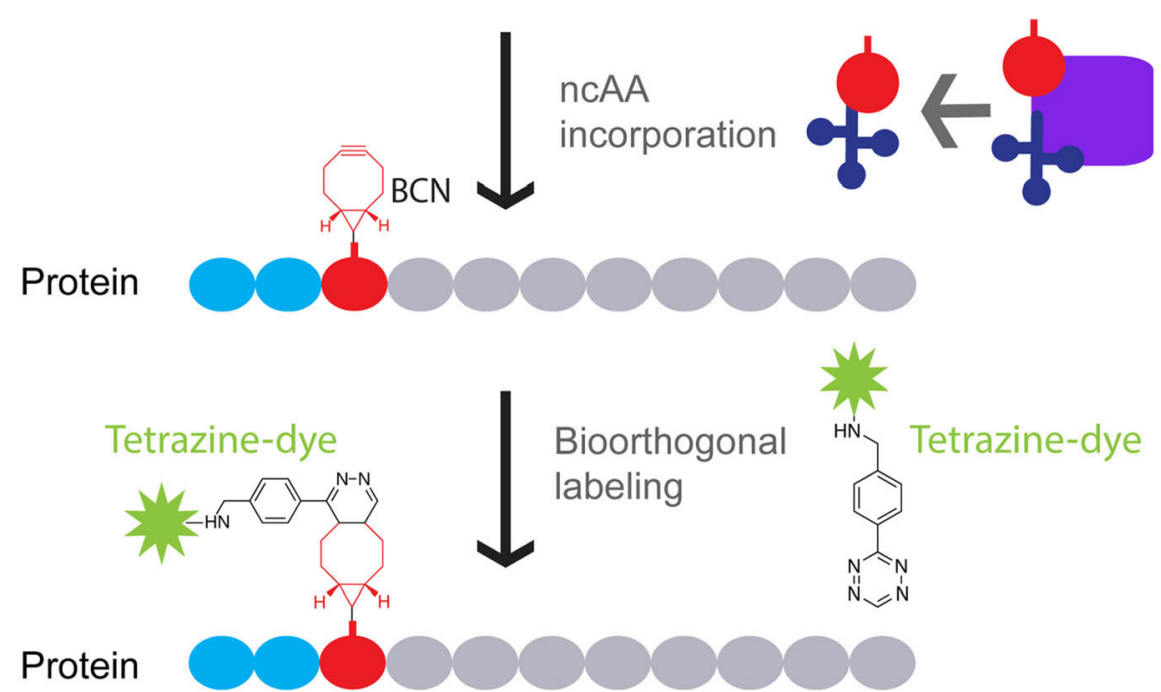

Fig. 1 Using a GCE-tag for fluorescence labeling of proteins via genetic code expansion (GCE) and bioorthogonal chemistry. a Schematic representation of the labeling approach. A GCE-tag (blue) is attached to the N-terminal of the protein (gray). Binding of the Fl-dye (green) to the GCE-tag leads to labeling of the protein. $\mathbf{b}$ Graphical illustration of the experimental design. The stop codon, UAG, and nucleotides encoding a short polypeptide tag are added upstream to the coding sequence of a protein. During ribosomal translation, the nCAA BCN-Lys (red) is incorporated into the protein in response to the in-frame UAG codon using a specific, orthogonal pair of tRNA (dark blue)/tRNA-Synth (purple). Finally, a tetrazine-conjugated Fl-dye (green) is covalently attached to BCN-Lys via a bioorthogonal reaction. As a result, the protein is directly labeled with Fl-dye via a small polypeptide tag. 
cloned into a single expression vector, designed to encode the incorporation of the ncAA bicyclo-nonyne Lysine (BCN-Lys) that bioorthogonally reacts with tetrazineconjugated Fl-dyes (Fig. 1b and Additional file 1: Figure S1a) $[6,18,19]$. Labeling potency was initially assessed using $\alpha$-tubulin as a benchmark and evaluating microtubule (MT) labeling in live mammalian cells in the presence of Silicon-Rhodamine-Tetrazine (SiR-Tet) [6, 20]. MT labeling obtained upon site-specific incorporation of BCN-Lys in $\alpha$ tubulin at position 45 ( $\alpha$-tubulin ${ }^{45 T A G}$ ) was used as a reference, given our earlier demonstration of the efficacy of MT labeling at this site [6].

The most minimal N-terminal GCE-tag should include a TAG codon that will encode the incorporation of BCN-Lys. Unfortunately, no specific labeling was obtained upon expressing $\alpha$-tubulin that carries a TAG codon at its $\mathrm{N}$-terminal (directly before the first residue) in the presence of SiR-Tet, using live-cell imaging or ingel fluorescence (Additional file 1: Figure S1b and S2). This indicates that the sole addition of a TAG codon upstream of a protein coding sequence is not sufficient for labeling, perhaps due to the failure of the ribosome to incorporate the ncAA at the very N-terminal of a protein. Potential N-terminal tags, bearing different lengths, were therefore designed based on the commonly used nine amino-acid (AA)-long hemagglutinin (HA) epitope as a backbone [21] (Fig. 2a and Additional file 1: Figure S1c-f). The TAG codon was either introduced within the HA sequence (replacing the last codon in the epitope) (1), immediately after the HA sequence (2), or after a short commonly used flexible linker comprising either glycine-serine $(G S ; 3)$ or glycine-glycine-serine-glycine (GGSG; 4). Low but noticeable levels of $\alpha$-tubulin were observed using any of these tags, indicating that $\mathrm{N}$ terminally tagged $\alpha$-tubulin was expressed in cells (Fig. 2b). In live-cell labeling experiments with SiR-Tet, little to no MT decoration was obtained using $\alpha$-tubulin tagged with probes 1 or 2 (Fig. 2c, 1-2), while clear and specific labeling was observed using tags 3 and 4 (Fig. 2c, $3-4)$, indicating that probes 3 and 4 can potentially serve as GCE-tags for $\alpha$-tubulin.

To evaluate labeling efficiencies obtained using potential GCE-tags, signal-to-noise ratios (SNRs) were measured in labeled cells based on line intensity profiles, as illustrated in Fig. 2d. SNR values measured in cells expressing any of the $\alpha$-tubulin versions were significantly higher than the average background levels measured in cells expressing a wild type (WT) version of $\alpha$-tubulin that is unable to incorporate the ncAA, verifying that labeling is specific (Fig. 2e). Cells expressing $\alpha$-tubulin tagged with probe 4 exhibited significantly higher SNR compared to probe 3 , indicating that HA-GGSG-ncAA is preferable over HA-GS-ncAA for MT labeling. Notably, despite the relatively low expression levels obtained using probe 4 , the average SNR values obtained in cells expressing $\alpha$-tubulin tagged with probe 4 were higher than those obtained in cells expressing $\alpha$ tubulin ${ }^{45 T A G}$ or in cells expressing $\alpha$-tubulin-GFP (Fig. 2d, e) [6]. Improved SNRs under lower $\alpha$-tubulin expression levels can result from reducing the fraction of cytosolic (non-polymerized)-labeled tubulin in cells and is preferential for cell physiology. We therefore concluded that HA-GGSG-ncAA-tagged $\alpha$-tubulin is superior to site-specific ncAA-incorporated $\alpha$-tubulin for MT labeling. Based on these results we defined the $\mathrm{N}^{\prime} \mathrm{HA}$ GGSG-ncAA as the minimal tag for GCE-based bioorthogonal labeling of proteins in live mammalian cells (Fig. 2f).

To examine the applicability and specificity of the GCEtag (HA-GGSG-ncAA, Fig. 2f and Additional file 1: Figure S1f) for labeling different cellular compartments, we first applied it to cellular markers that are conjugated to GFP, i.e., the plasma membrane (PM) marker GFP-CAAX and the peroxisomal marker GFP-SKL (Figs. 3 and 4). GCEtagged GFP-CAAX was expressed in cells at higher levels than GFP-CAAX that carries a TAG codon at the optimized position in GFP (GFP ${ }^{150 T A G}$-CAAX, Fig. 3a) [19]. Consistently, PM decoration was observed in cells expressing either GCE-tagged GFP-CAAX or GFP ${ }^{150 T A G}$-CAAX and labeled with SiR-Tet in both the GFP and SiR channels with relatively similar SNR values (Fig. 3b, c). SNR values measured for PM labeling using the SiR channel for GCE-tagged GFP-CAAX or GFP ${ }^{150 T A G}$ CAAX were similar and significantly higher than the levels measured in cells expressing GFP-CAAX that is unable to incorporate the ncAA (WT GFP-CAAX), indicating that labeling is specific and is not compromised by the GCE-tag (Fig. 3d, e).

GCE-tagged GFP-SKL was expressed in HEK293 cells, and a specific band at a similar size was observed in an in-gel fluorescence assay upon labeling the cells with SiR-Tet, in the presence of BCN-Lys (Fig. 4a, b). Notably, while WT GFP-SKL was expressed at higher levels compared to GCE-tagged GFP-SKL, no specific labeling was observed for WT GFP-SKL using in-gel fluorescence, strongly indicating that labeling is specifically induced by binding of the Fl-dye to the ncAA in GCEtagged GFP-SKL. In live cells, remarkable co-localization of GFP and SiR in small puncta, was observed throughout the cell (except in the nucleus) at similar SNRs (Fig. 4c, Pearson's correlation $=0.86$ ). This was not the case in cells expressing WT GFP-SKL and labeled with $\mathrm{SiR}$, which exhibited an anti-correlation between GFP and SiR puncta (Fig. 4d, e, Pearson's correlation $=0.07$ ) In these cells, a small population of SiR puncta was observed but these puncta did not co-localize with the GFP puncta observed throughout the cell. These results further indicate that the co-localization observed in cells 
a

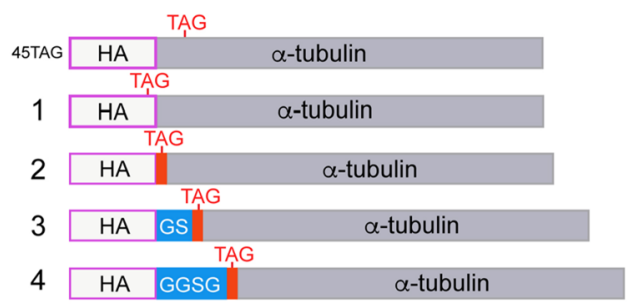

b

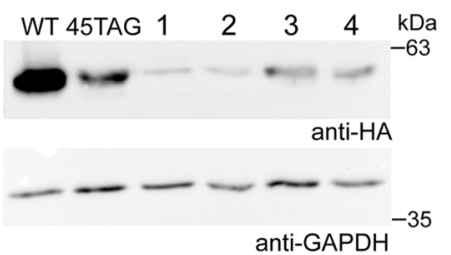

C
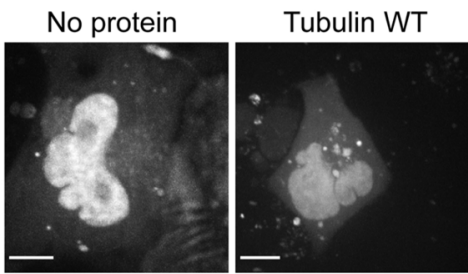

Tubulin 45TAG

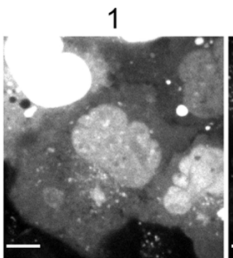

2
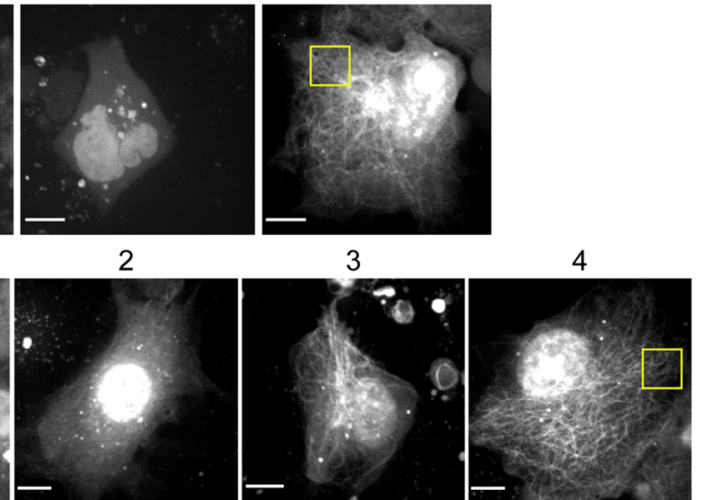

d
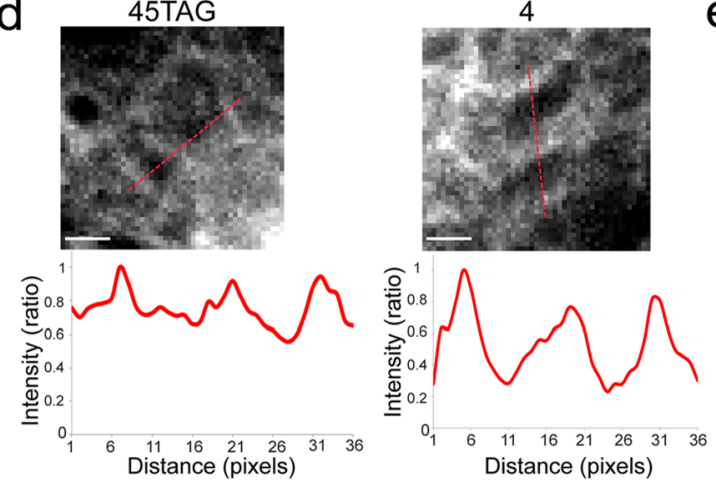

e

f

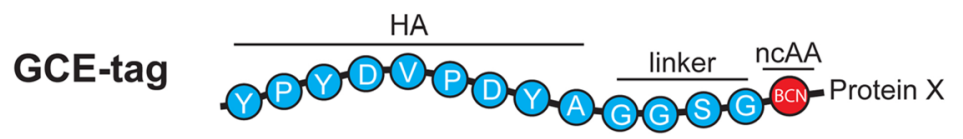

Fig. 2 Optimizing a minimal tag for genetic code expansion (GCE)-based fluorescence labeling using a-tubulin. a Schematic view of the tags used and tested in $\mathbf{b}$-e. Complete sequences of the tags are shown in Additional file 1: Figure S1c-f. b-e GCE-tags (specified in a) were subcloned to the N-terminal of a-tubulin using the pBUD-Pyl-RS-tub plasmid (Additional file 1: Figure S1a). HEK293T (b) or COS7 (c-e) cells were transfected with pBUD-PyI-RS-tub plasmids carrying different tags, a WT version of a-tubulin, or without a target protein, and incubated for $48 \mathrm{~h}$ in the presence of the nCAA BCN-Lys. Cells were then subjected to western blot analysis (b) or labeled with SiR-Tet for $1 \mathrm{~h}$ and imaged live (c-e). Images in $\mathbf{c}$ are maximum intensity projections of 3D z-stacks obtained from representative cells. $\mathbf{d}$ Zoomed-in images of the regions marked in yellow squares in c. Intensity values along the red lines drawn in the images are plotted below, demonstrating the improvement in SNR. e SNR values measured in cells expressing a-tubulin with BCN-Lys incorporated either in position G45 or in the N-terminal via tags 3 or 4 . The averaged SNR measured in cells expressing WT tubulin was 1.2 (red dashed line). The averaged SNR previously measured using GFP was 1.75 (green dashed line). $n=25$. Results presented in $\mathbf{b}$-e were obtained in at least 3 independent experiments. $\mathbf{f}$ Schematic view of the optimized GCE-tag used for further labeling. Scale bars: c $10 \mu \mathrm{m}, \mathbf{d} 2 \mu \mathrm{m}$

expressing GCE-tagged GFP-SKL resulted from specific SiR labeling of the protein.

The organization of the fluorescence signal into discrete puncta in SKL labeled cells allowed us to estimate relative labeling yields by segmenting the population of puncta in one channel and quantifying their intensities in the other channel (Fig. 4f, g). GFP puncta segmented from cells expressing WT GFP-SKL and labeled with SiR-Tet showed low intensity SiR levels that did not scale with GFP intensity levels (Fig. 4f). We 


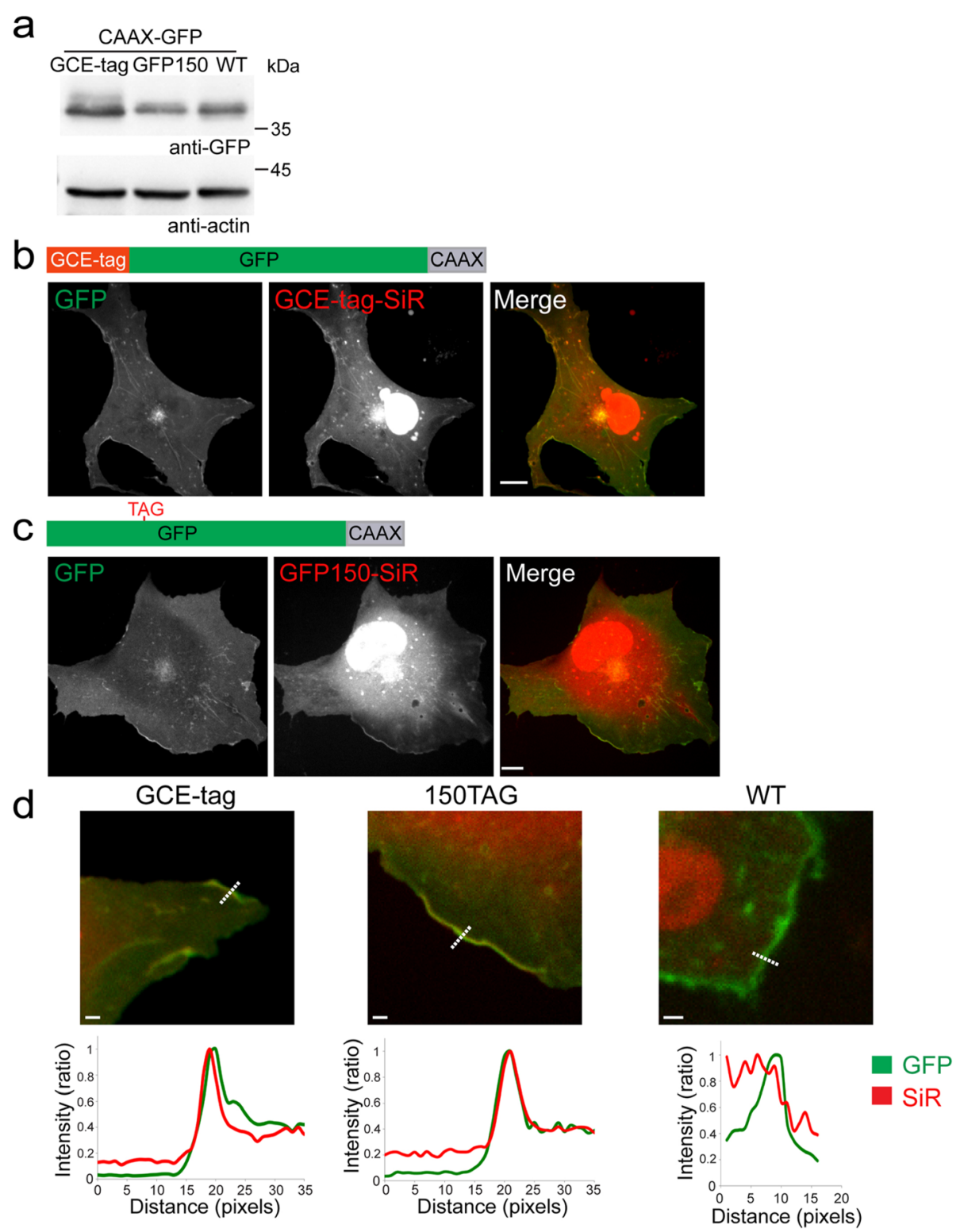

e

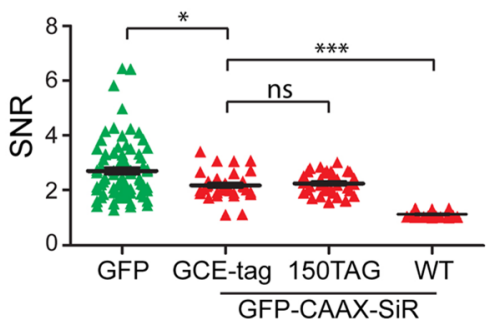

Fig. 3 Plasma membrane (PM) labeling obtained via the minimal GCE-tag is comparable to GFP and site-specific labeling. HEK293T (a) or COS7 (b-e) cells were transfected with pBUD-Pyl-RS plasmids carrying GCE-tag-GFP-CAAX, GFP-CAAX mutated at position 150 to TAG or a WT version of GFP-CAAX, and incubated for $48 \mathrm{~h}$ in the presence of BCN-Lys. Cells were then subjected to western blot analysis (a) or labeled with SiR-Tet for $1 \mathrm{~h}$ and imaged live (b-e). Images represent single confocal slices of representative cells. $\mathbf{d}$ Line intensity profiles obtained in cells expressing the indicated constructs and labeled with SiR-Tet. Upper panel: zoomed-in images of confocal slices. Bottom panel: intensity values along the dashed line drawn in the images. e SNR values measured for GFP and SiR in cells expressing the different versions of GFP-CAAX. $n=30$. Results were obtained in at least 3 independent experiments. Scale bars: b, c $10 \mu \mathrm{m}, \mathbf{d} 2 \mu \mathrm{m}$ 
a

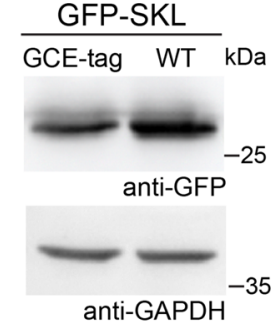

C GCE-tag

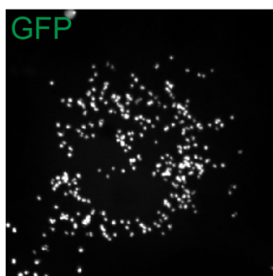

d

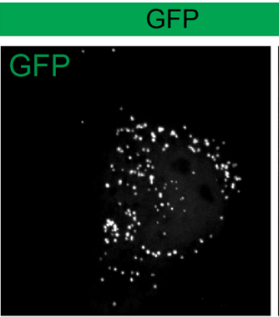

e

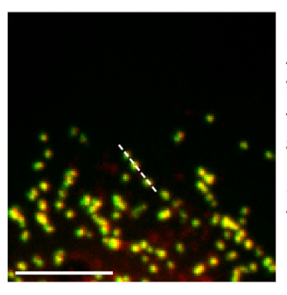

f

$20000 \bigcirc$ GCE-tag GFP-SKL

$\overbrace{}^{16000}$ WT GFP-SKL

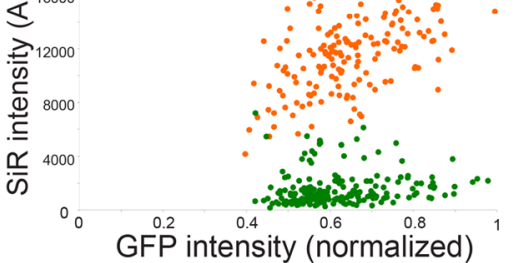

GFP intensity (normalized)

SKL

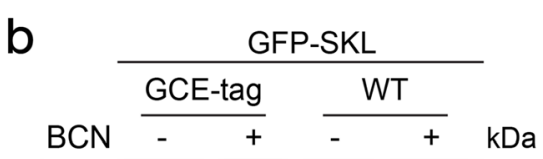

$-25$

SKL
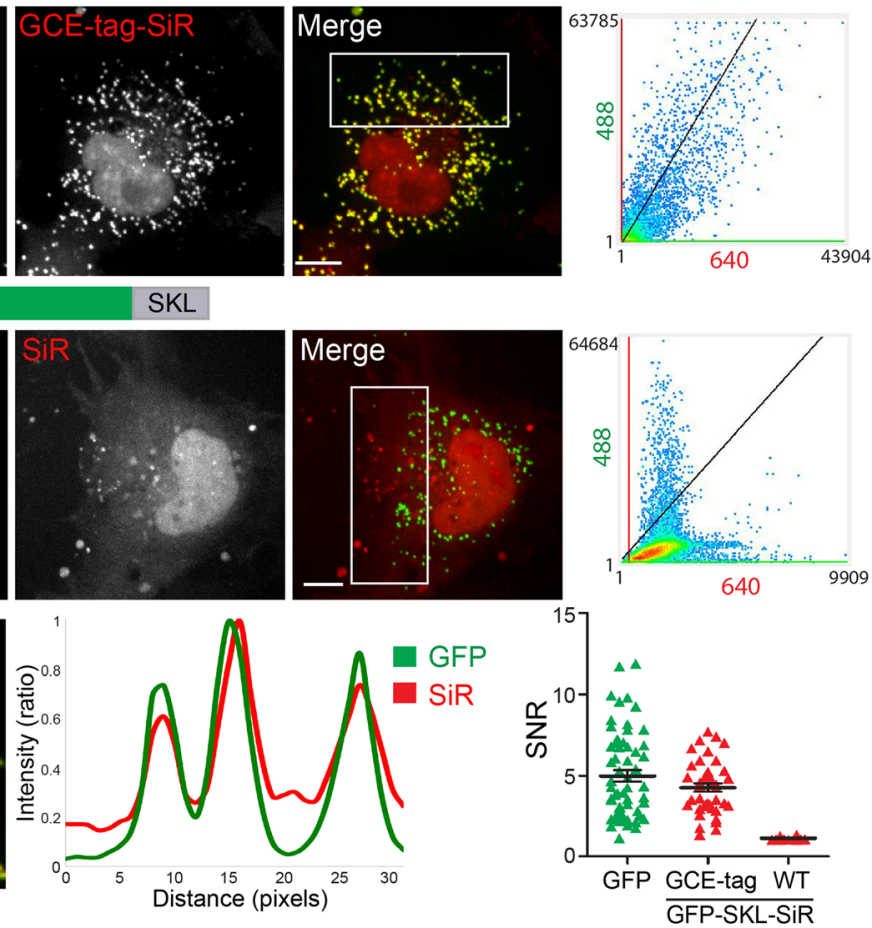

9 ${ }^{50000} \bigcirc$ GCE-tag GFP-SKL

$\widehat{\}_{40000}^{45000}$ WT GFP-SKL

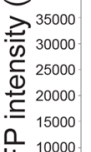

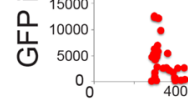

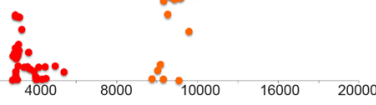

SiR intensity (AU)

Fig. 4 (See legend on next page.) 
(See figure on previous page.)

Fig. 4 Demonstrating labeling specificity and efficiency in peroxisomes labeled with GCE-tagged GFP-SKL. HEK293T (a, b) or COS7 (c-f) cells were transfected with pBUD-Pyl-RS plasmids carrying the indicated GFP-SKL constructs and incubated for $48 \mathrm{~h}$ in the presence of the nCAA BCN-Lys (except for lanes 1 and 3 in $\mathbf{b}$ ). Cells were then subjected to western blot analysis (a) or labeled with SiR-Tet for $1 \mathrm{~h}$ and subjected to in-gel fluorescence (b) or imaged live $(\mathbf{c}-\mathbf{g})$. b Cells were lysed, and equal total protein amounts were loaded on an SDS-PAGE. Results were recorded using SiR fluorescence. A specific band obtaining a similar size to that obtained in western blot was seen only in cells expressing GCE-tag-GFPSKL and incubated with BCN-Lys, indicating specific labeling. c, d Maximum intensity projections of representative cells. Left to right: fluorescence obtained in each channel, merged image and co-localization analysis between intensity values of the 640 (SiR) and 488 (GFP) on a large subset of the cell that excludes the nucleus (white rectangle in merged image): Pearson's correlation values: c 0.86, d 0.07. e Left to right: a zoomed-in image of the cell in $\mathbf{c}$, a line intensity profile measured for the dashed line in the zoomed-in image, and a summary of SNR values measured for GFP and SiR in cells expressing the different versions of GFP-SKL. $n=40 . \mathbf{f}, \mathbf{g}$ Cells expressing the indicated constructs were labeled with SiR-Tet and imaged using similar imaging parameters (exposure time and laser intensity). $\mathbf{f}$ GFP-positive peroxisomes were segmented based on intensity. For each peroxisome, the mean intensity in the GFP channel was plotted against the mean intensity in the SiR channel. 95\% of GFPlabeled peroxisomes exhibited SiR intensity levels that are above background. $\mathbf{g}$ SiR-positive puncta were segmented based on intensity. For each punctum, the mean intensity in the SiR channel was plotted against the mean intensity in the GFP channel. 5\% of SiR-positive puncta were GFP negative. Mean intensity levels measured for SiR puncta in cells expressing GCE-tag-GFP-SKL were at least twofolds higher than the levels measured for SiR puncta segmented in control cells expressing WT GFP-SKL, indicating specific labeling. Results were obtained in at least 3 independent experiments. Scale-bars $10 \mu \mathrm{m}$

therefore reasoned that the SiR levels obtained in these cells represent background SiR fluorescence. In cells expressing GCE-tagged GFP-SKL, the majority of segmented GFP puncta $(\sim 95 \%)$ had higher SiR intensity levels than those measured in cells expressing WT GFPSKL. Moreover, intensity levels in the SiR channel scaled with GFP intensities, indicating that SiR fluorescence can be used for quantifying the relative levels of GFPSKL in single peroxisomes. Segmenting the SiR-positive puncta in cells expressing GCE-tagged GFP-SKL globally resulted in similar behavior, with a small population (less than $5 \%$ ) of SiR puncta that appeared GFP negative (Fig. 4g). In cells expressing WT GFP-SKL, a small population of SiR puncta was segmented. These puncta had low intensity levels in both GFP and SiR channels compared to SiR puncta segmented in GCE-tagged GFPSKL expressing cells, indicating that they represent noise. These structures can be easily filtered out based on intensity, using image processing. Taken together, these data indicate that GCE-tag SiR-based labeling has $\sim 95 \%$ yield compared to GFP and that it can be used for quantitative live-cell imaging of peroxisomes.

Next, we tested the robustness of the tag by applying it to diverse cellular organelles. For that, we used Lamp1 as a lysosomal marker, CD63 as a marker of multivesicular bodies (MVBs), $\mathrm{ER}^{\mathrm{cb} 5} \mathrm{TM}$ as an endoplasmic reticulum (ER) marker, Exo70 as an exosomal marker, and Mito ${ }^{\text {cb5 }} \mathrm{TM}$ (mito) or mito-DsRed as mitochondrial markers (for sequences see Additional file 1: Table S1). Nuclear labeling was avoided due to the known nonspecific labeling of the nucleus observed with GCE-based labeling (Figs. 2c; 3b, c; and 4c, d) [4, 6, 16]. Effective lysosome labeling was obtained using GCE-tagged Lamp1 in the presence of SiR-Tet (Fig. 5a, b). Overall, labeling was comparable to that obtained with Lamp1-mVenus. Lysosomes labeled via either of the tags (i.e., GCE-tag or mVenus) exhibited a similar response to chloroquine treatment and had similar SNR levels (Fig. 5a-c) [22]. We noticed that while lysosome labeling using Lamp1mVenus highlighted both filled and hollow structures, only filled structures were observed in cells labeled with GCE-tag-SiR-Lamp1. This difference may result from the position of the tag; the mVenus tag faces the cytosol, while the GCE-tag faces the lysosomal lumen. Labeling lysosomal lumen proteins using conventional fluorescent proteins is challenging due to the $\mathrm{pH}$ sensitivity of the latter [23]. Our data suggest that GCE-tag-based labeling is compatible with labeling acidic cellular structures.

Initial attempts at labeling MVBs, ER, mitochondria, and exosomes using the GCE-tag and SiR-Tet resulted in no specific staining (Additional file 1: Figure S3). Yet, in immunostaining experiments using anti-CD63 antibodies, GCE-tagged CD63 co-localized with endogenous CD63 (Fig. 5d). We thus reasoned that GCE-tagged CD63 was properly expressed and targeted in cells, yet failed to bind the Fl-dye via the bioorthogonal reaction. Consistent with this notion, substituting TAMRA-Tet for SiR-Tet resulted in specific labeling of MVBs, ER, and exosomes using GCE-tagged CD63, ER ${ }^{\mathrm{cb} 5} \mathrm{TM}$, and Exo70, respectively (Figs. 5e-g and 6). Mitochondrial labeling was not obtained using either SiR- or TAMRA-Tet together with the GCE-tag-Mito ${ }^{\mathrm{cb} 5} \mathrm{TM}$ or mito-DsRed probes (Additional file 1: Figure S4). Based on immunofluorescence experiments, it appears that while $\mathrm{Mito}^{\mathrm{cb} 5} \mathrm{TM}$ failed to localize to mitochondria, GCE-tagged mito-DsRed localized properly but failed to bind the tetrazine-conjugated dye (Additional file 1: Figure S4). Expression patterns and SNR values obtained with the GCE-tagged organelle markers that were successfully labeled (i.e., for MVBs, ER, and exosomes) were overall comparable to those obtained using conventional Fl-protein markers with better SNRs obtained for GCE-tagged MVBs and lower SNRs obtained for GCE-tagged ER (Figs. 5e-g and 6). In all cases (SiR- or TAMRA-labeled GCE-tagged cellular structures), signal 


\section{a}

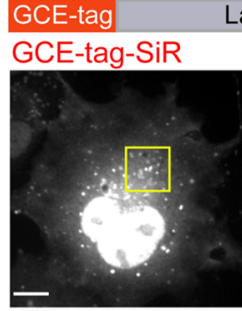

Lamp1
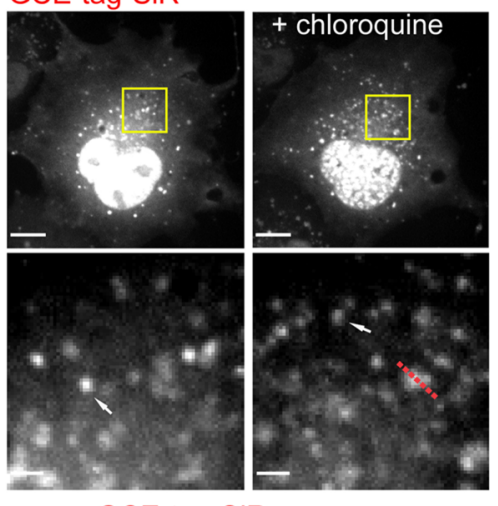

C
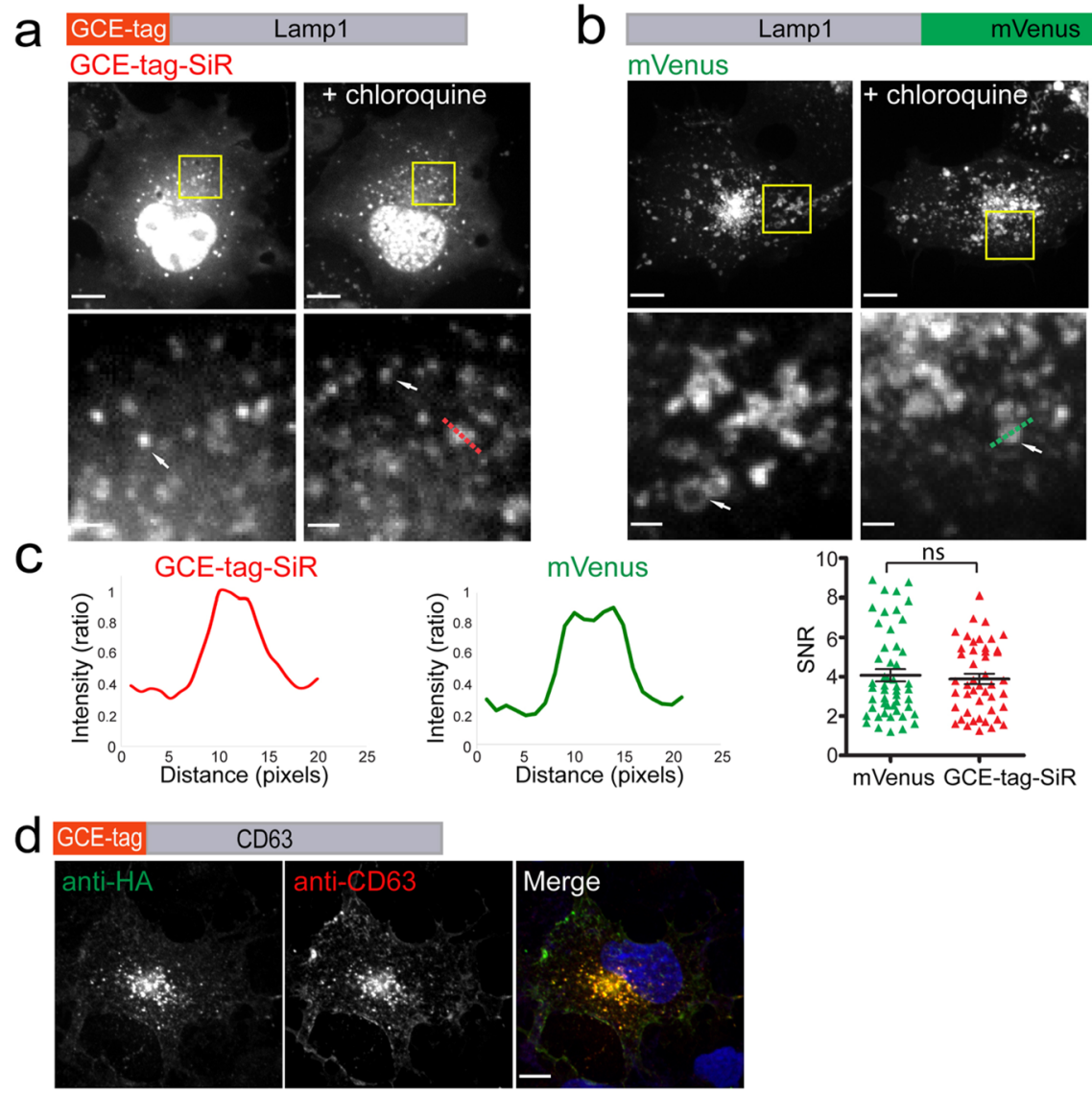

e GCE-tag CD63
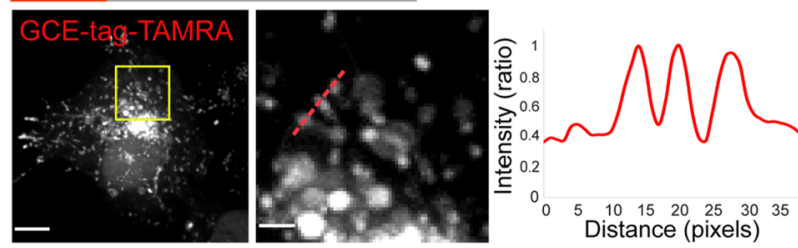

f

CD63
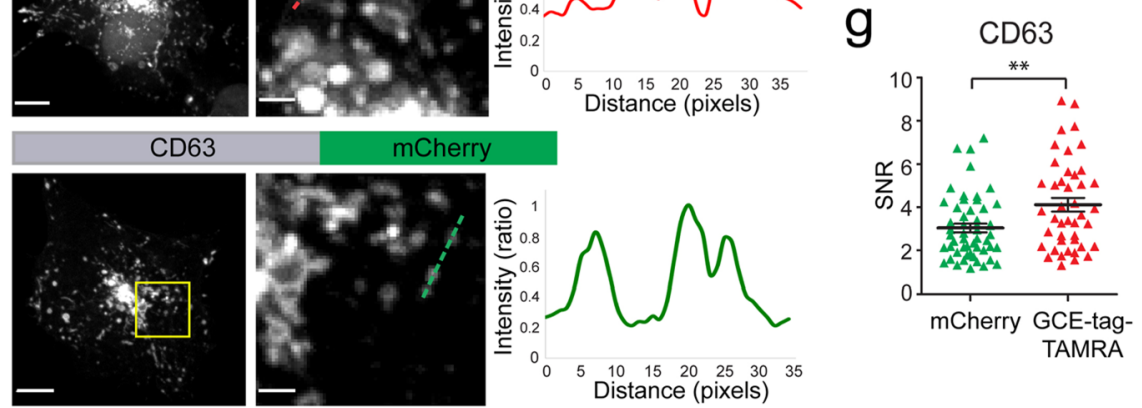

Fig. 5 Efficient lysosome labeling is obtained using SiR-Tet while MVB labeling requires TAMRA-Tet. a-c Cells expressing GCE-tag-Lamp1 labeled with SiR-Tet (a) or Lamp1-mVenus (b). Left panels: no treatment. Right panels: treatment with the lysosome inhibitor chloroquine $(3 \mathrm{~h}, 120 \mu \mathrm{M})$. Zoomed in images of a subset of the cells (correspond to squares in upper panels) are shown in bottom panels. Arrows indicate filled versus hollow lysosome labeling. $\mathbf{c}$ Left to right: line intensity profiles measured for the red dashed line in $\mathbf{a}$, the green dashed line in

$\mathbf{b}$, and a summary of SNR values measured in cells expressing the indicated plasmids $(n=50)$. $\mathbf{d}$ COS7 cells expressing the MVB marker CD63 conjugated to the GCE-tag were fixed and stained with anti-HA and anti-CD63 antibodies. e, f MVB labeling in cells expressing GCE-tag-CD63 labeled with TAMRA-Tet (e) or CD63-mCherry (f). $\mathbf{g}$ SNR values measured in cells expressing the indicated plasmids. $n=50$. Results presented in each panel were obtained in at least 3 independent experiments. Scale-bars, $10 \mu \mathrm{m}$; zoomed-in images, $2 \mu \mathrm{m}$

intensity levels were considerably higher than background levels measured in cells expressing a plasmid encoding only the orthogonal tRNA/tRNA-synthetase pair and labeled with the corresponding Tet-conjugated Fl-dyes (Fig. 6g). Together, these results validate labeling specificity and compatibility of the GCE-tag for organelle labeling. 


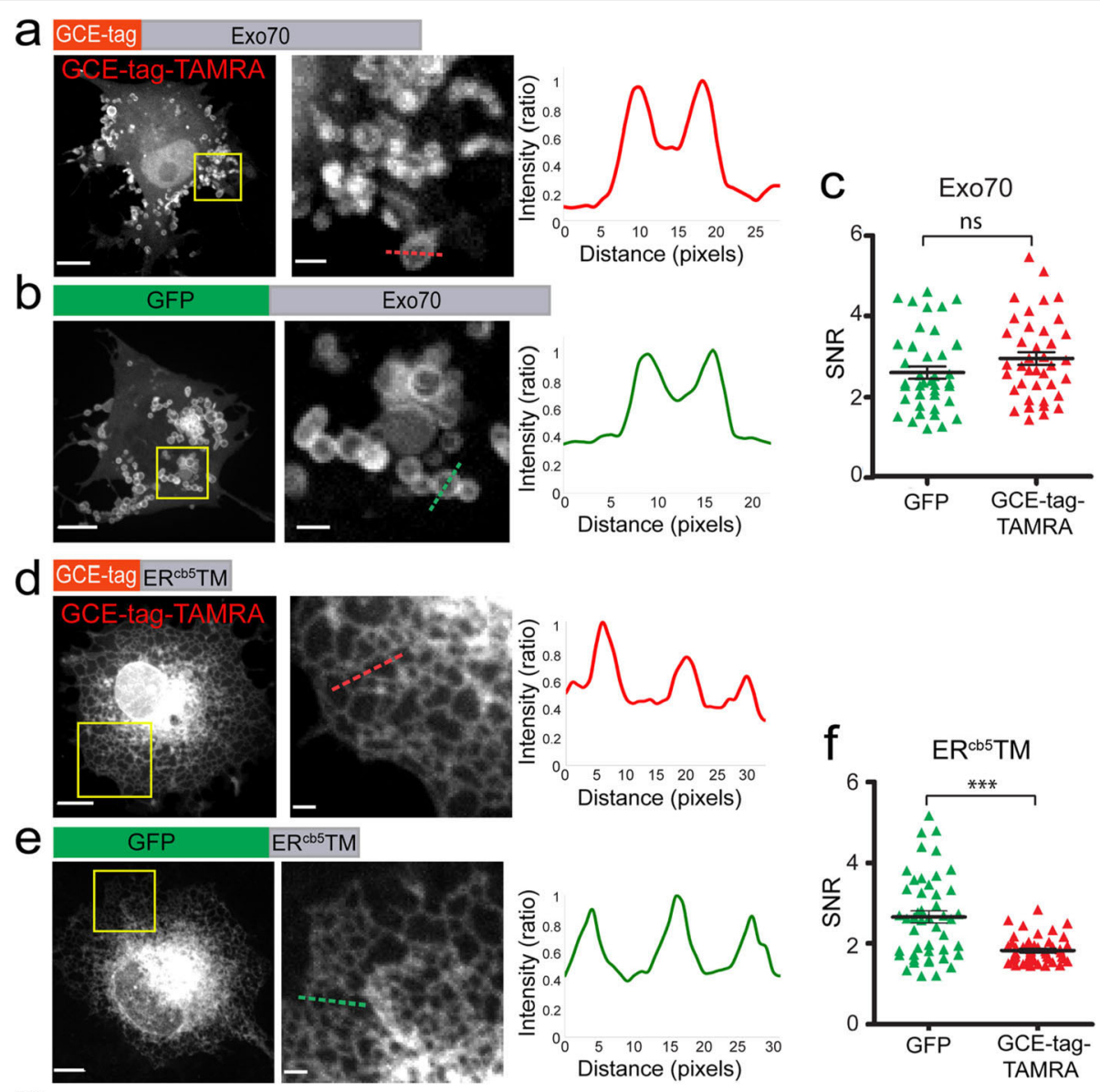

g

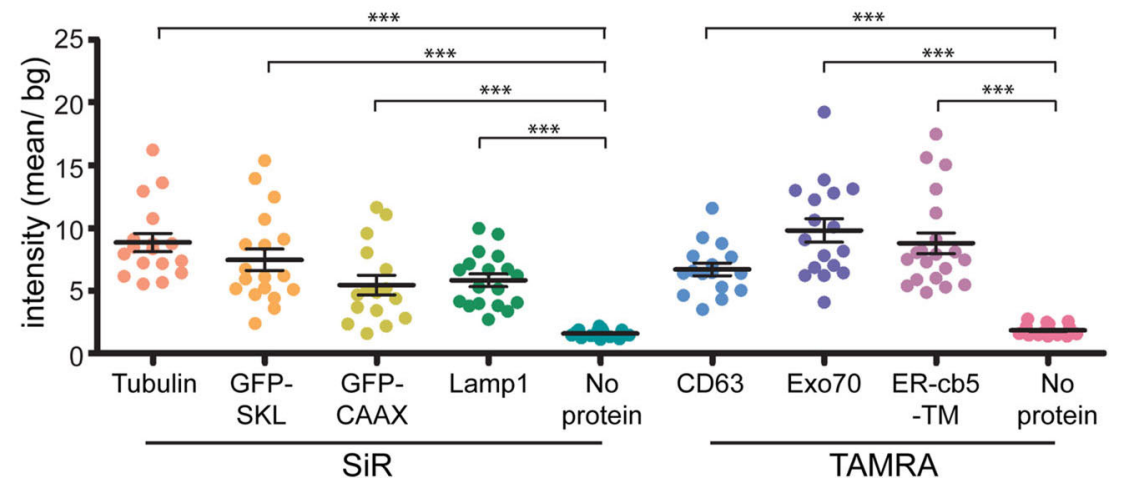

Fig. 6 Exosomes and ER exhibit specific cellular labeling via the minimal GCE-tag in the presence of TAMRA-Tet. COS7 cells were transfected with the indicated plasmids, labeled when indicated, and imaged live $48 \mathrm{~h}$ later. $\mathbf{a}, \mathbf{b}, \mathbf{d}$, e Left to right: maximum projection images, zoomed-in images of a subset of the cells (correspond to squares in left panels), line intensity profiles measured for the dashed lines in middle panels. c, $\mathbf{f} S N R$ values measured in cells expressing the indicated plasmids ( $n$ Exo70 $=40$, $E R^{\mathrm{cb} 5} T M=45$ ). $\mathbf{g}$ Cells were transfected with the indicated the pBUD-Pyl-RS plasmids and labeled with SiR-Tet or TAMRA-Tet, as indicated. For each condition, mean intensity levels were measured for a region of interest inside the cell and were divided by the mean background intensity measured outside the cell. pBUD-Pyl-RS plasmids that do not encode for a protein of interest were used as negative controls (no protein). $n=15$. Results presented in each panel were obtained in at least 3 independent experiments. Scale-bars, $10 \mu \mathrm{m}$; zoomed-in images, $2 \mu \mathrm{m}$

Successful and specific labeling was also obtained for GCE-tagged extracellular protein EGFR and intracellular ESCRT-III protein CHMP4B as indicated by in-gel fluorescence and live-cell imaging (Additional file 1:
Figure S5). For EGFR labeling, the GCE-tag was inserted between the signal peptide and the protein coding sequence (Additional file 1: Figure S5c, d), indicating that the GCE-tag is not exclusive for the N- 


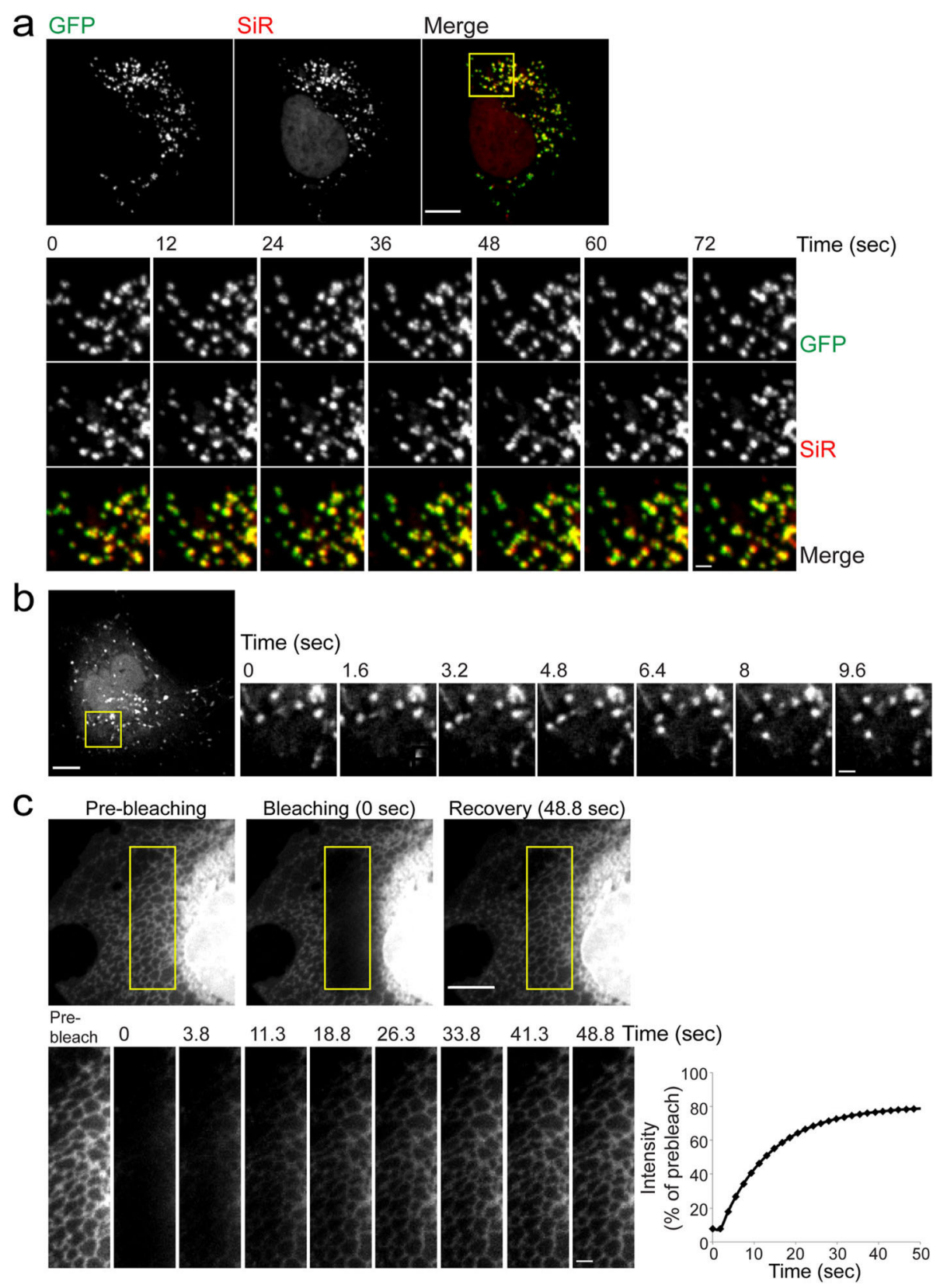

Fig. 7 Live-cell recordings of cellular organelles labeled with GCE-tag and a tetrazine-conjugated Fl-dye. a Live-cell recordings of cells expressing GCEtag-GFP-SKL labeled with SiR-Tet. Upper panel: maximum projection images of the entire cell taken at time 0. Bottom panel: sequential zoomed-in images of a subset of the cell (corresponds to square in upper panel) taken from the movie series. Every fourth frame from the movie series is shown (Additional file 4: Movie S3). b Live-cell recordings of cells expressing GCE-tag-CD63 labeled with TAMRA-Tet. Left, a maximum projection image of the entire cell taken at time 0 . Sequential zoomed-in images of a subset of the cell (corresponds to square in left panel) taken from the movie series are shown to the right. Every fourth frame from the movie series is shown (Additional file 6: Movie S5). c FRAP analysis of cells expressing GCE-tag-ER ${ }^{\mathrm{cb5}} \mathrm{TM}_{\mathrm{TM}}$ labeled with TAMRA-Tet. Snapshots of a subset of a representative cell taken from the movie series (Additional file 8: Movie S7) are shown in the upper panel. Sequential images of the bleached ROI are shown in the bottom panel (Additional file 9: Movie S8). Plot on the right represents the exponential fit of fluorescence intensity recovery versus time after photobleaching in the ROI. Intensity values were corrected for unintentional bleaching and normalized to intensity levels measured pre-bleaching. Results presented in each panel were obtained in at least 3 independent experiments. Scalebars, $10 \mu \mathrm{m}$; zoomed-in images, 2 mm 
terminal. Thus, besides its use in organelle labeling, the GCE-tag can be used for labeling intra- and extracellular proteins in live cells.

Fl-dye labeling of the GCE-tagged constructs allowed live-cell recordings and tracking of any of the labeled cellular structures (i.e., MT, PM, peroxisomes, ER, MVB, and exosomes; Fig. 7 and Additional file 2: Movie S1, Additional file 3: Movie S2, Additional file 4: Movie S3, Additional file 5: Movie S4, Additional file 6: Movie S5, Additional file 7: Movie S6, Additional file 8: Movie S7, and Additional file 9 Movie S8). Fluorescence recovery after photobleaching (FRAP) experiments were successfully performed using the GCE-ER ${ }^{\mathrm{cb} 5} \mathrm{TM}$ ER marker, with comparable recovery times to those measured in the ER using VSVG-GFP [24] (Fig. 7c and Additional file 8: Movie S7, Additional file 9: Movie S8). It is worth mentioning that the overall size of the GCE-tag-ER ${ }^{\mathrm{cb} 5} \mathrm{TM}$ ER marker is considerably smaller than VSVG-GFP (GCEtag-ER ${ }^{\mathrm{cb} 5} \mathrm{TM}, \sim 8.5 \mathrm{kDa}$; VSVG-GFP, $\sim 84.5 \mathrm{kDa}$ ). Taken together, these results indicate that the newly developed GCE-based organelle markers reported here can be employed to study organelle dynamics in live cells with much smaller probes.

The 14-residue long GCE-tag described here comprises the complete sequence of the HA tag. As such, the HA epitope can still be exploited for other applications, such as immunoblot, immunofluorescence, and immunoprecipitation (Figs. $2 \mathrm{~b}$ and $5 \mathrm{~d}$ ). To expand the versatility of the tag, we tested whether the HA epitope can be replaced by FLAG (8 residues) or Myc (10 residues) epitopes (Fig. 8 and Additional file 1: Figure S6). GFP-SKL constructs carrying HA/FLAG/Myc GCE-tags were successfully expressed in cells, with SiR-Tet fluorescence co-localizing with GFP (Pearson's correlation values: FLAG, 0.87; Myc, 0.67) (Fig. 8a, b upper panel, and Additional file 1: Figure S6a, b). Efficient labeling of exosomes was also obtained with the FLAG and Myc tag versions conjugated to Exo70, as indicated by live-cell imaging and in-gel TAMRA fluorescence (Fig. 8c upper panel and Additional file 1: Figure S6c, e). MT labeling, however, appeared to be compromised using FLAG/Myc GCE-tags, with very few cells exhibiting MT staining using the Myc GCE-tag (Fig. 8d upper panel and Additional file 1: Figure S6d). Reduced specific labeling was also observed using in-gel SiR fluorescence (Additional file 1: Figure S6f). However, while MT staining was more prominent using FLAG GCE-tag, higher labeling was observed for Myc GCE-tag using in-gel fluorescence. This data suggests that in some cases, the total level of labeled protein does not reflect its ability to label a specific cellular structure. Collectively, these results demonstrate that the system is versatile, in terms of the epitope used in the tag. But, while the HA version of the tag appears to be robust, labeling using the other epitope versions needs to be evaluated more carefully.

Last, we tested how removing the epitope sequence from the GCE-tag will affect labeling (Additional file 1: Figure S1i). In other words, is a sequence encoding GGSG linker followed by a TAG is sufficient for GCEbased bioorthogonal labeling of proteins? Almost no specific labeling was obtained upon tagging $\alpha$-tubulin with a GCE-tag that lacks the HA sequence both by ingel fluorescence and live-cell imaging (Fig. 8d bottom panel and Additional file 1: Figure S6f). For peroxisomes and exosomes, specific labeling was obtained, albeit at reduced levels (Fig. 8b, c bottom panels). In peroxisomes, the reduced labeling was consistent with the reduced expression of the protein observed by western blot (Fig. 8a, b bottom panel; Pearson's correlations, 0.76). Reduced specific labeling was also observed for Exo70 using in-gel fluorescence (Additional file 1: Figure S6e), raising the possibility that the reduced labeling observed in cells is a result of failure to incorporate the ncAA during translation. Therefore, in specific cases in which the length of the tag is critical for preserving the function of the protein, the GCE-tag may be reduced to as few as five residues. Yet, this comes at the price of labeling efficiency and thus should be tested on a caseby-case basis.

\section{Discussion}

In this work, we present a generic, small tag for labeling proteins in live mammalian cells with Fl-dyes through GCE and bioorthogonal chemistry, using a single expression vector. Efficient and specific labeling with Fl-dyes was observed for various intracellular structures and compartments, including MTs, PM, exosomes, lysosomes, MVBs, peroxisomes, and ER using appropriate tag-bearing markers, and for extracellular and cytosolic proteins that carry the tag. By adding 14 residues to the $\mathrm{N}$-terminal of proteins, we minimized the need for prior knowledge on the protein and bypassed the screening step currently associated with the technique. Moreover, by inserting the TAG codon at the beginning of the coding sequence (rather than in the middle of the sequence), we avoided the expression of truncated proteins. As labeling efficiencies obtained using the GCE-tag were either comparable or superior to sitespecific labeling, the GCE-tag presented here provide an attractive, easy-to-implement, alternative for bioorthogonal labeling of proteins modified to carry a ncAA.

The GCE-tag reported here is considerably smaller than Fl-protein tags and self-labeling protein tags (GCE-tag, 1.5 kDa; GFP, $27 \mathrm{kDa}$; SNAP-tag, $19 \mathrm{kDa}$ ) $[1,2]$. The only tag with a comparable size to that of the GCE-tag is the 12 amino-acid-long FlAsH tag 
a

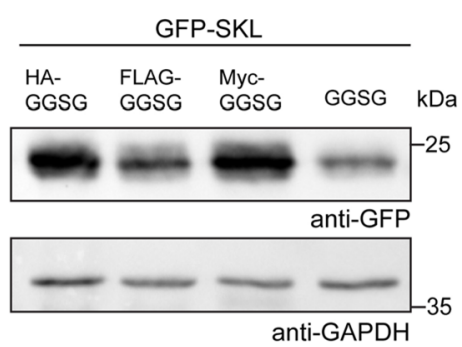

b FLAG GGSG TAG GFF
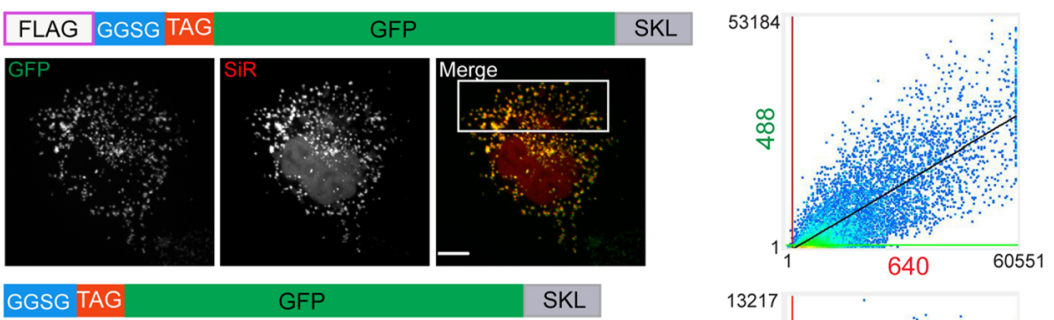

GFP

SKL
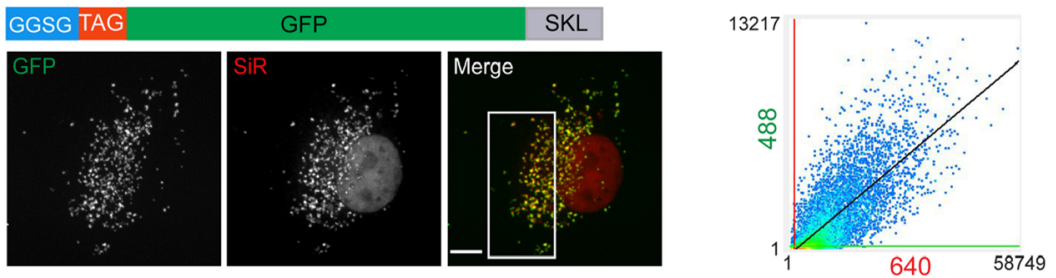

C FLAG GGSG TAG protein
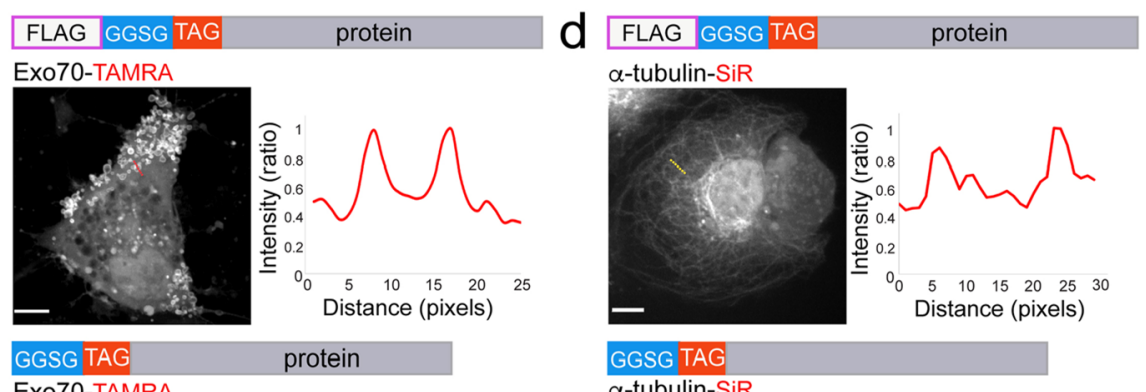

Exo70-TAMRA
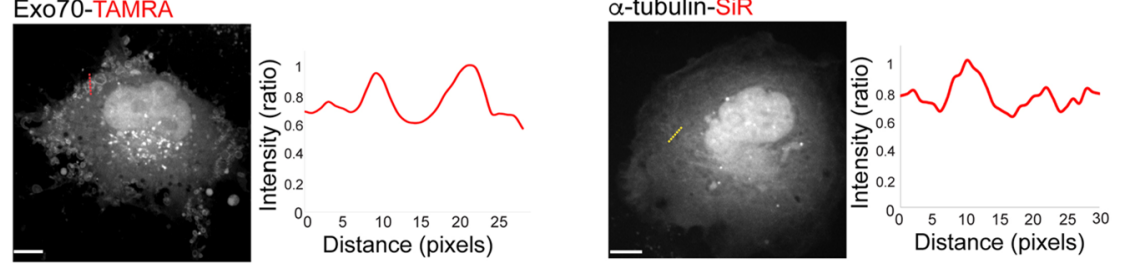

Fig. 8 The GCE-tag is versatile and in some cases can be further minimized. a Western blot analysis of HEK293T cells transfected with pBUD-Pyl-RS plasmid inserted with GFP-SKL N-terminally tagged with (from left to right) the optimized GCE-tag (HA-GGSG-ncAA), tags with alternative short epitopes FLAG-GGSG-nCAA and Myc-GGSG-ncAA, and a tag with no epitope (GGSG-ncAA). Cells were incubated for $48 \mathrm{~h}$ in the presence of BCN-Lys, harvested and subjected to western blot analysis using anti-GFP antibodies. b-d Maximum intensity projections taken from live-cell images of COS7 cells transfected with FLAG-GGSG-ncAA (upper panels) or GGSG-ncAA (lower panels) conjugated to GFP-SKL (b), Exo70 (c), or a-tubulin (d) and labeled with the indicated tetrazine dyes. Plots in $\mathbf{b}$ represent co-localization analysis between intensity values of the 640 (SiR) and 488 (GFP) channels performed on large subsets of the cells that exclude the nucleus (correspond to rectangles in merged image); Pearson's correlation values: upper panel, 0.87; bottom panel, 0.76. c, $\mathbf{d}$ Left, maximum intensity projection of representative cells. Right, line intensity profiles measured for the dashed lines in left panels. Results presented in each panel were obtained in at least 3 independent experiments. Scale bar $10 \mu \mathrm{m}$

[25]. However, labeling proteins with FlAsH tags rely on biarsenical-functionalized fluorescent dyes, which exhibit unspecific binding to cellular membranes and are toxic to cells [2]. Therefore, although adding a tag is not as elegant as site-specific labeling, the GCE-tag stands as one of the shortest tags available for fluorescence labeling of proteins and organelles in live cells.
Using GCE-based bioorthogonal labeling is known to suffer from relatively high noise levels compared to fluorescent protein tag-based approaches. The main noise sources are non-specific binding of the tetrazine-conjugated fluorescent dyes and excess of tRNA that is charged with a ncAA and is free to undergo the bioorthogonal reaction. The latter is the main source for the unspecific staining observed in the 
nucleolus. To minimize these noise factors, we previously performed careful optimization of all assay components $[6,19]$. Using our optimized labeling assay conditions, GCE-tag-based labeling was comparable to protein tags in terms of labeling efficiencies and SNR values. Given the small size of the tag, which is likely to better preserve the physiological properties of proteins and organelles, we find GCE-tag superior to conventional protein tags for protein labeling.

While demonstrating the suitability of the GCE-tag for labeling proteins in a variety of cellular compartments, the results described here stress the need to test several dyes when calibrating conditions for bioorthogonal labeling. Table 1, summarizing the dyes found suitable for labeling each organelle, can be used as a guideline when optimizing labeling conditions for different cellular proteins. The increasing number of commercially available tetrazine-conjugated dyes will no doubt expand the ability to tailor Fl-dyes to specific cellular environments.

Successful GCE-based bioorthogonal labeling mainly relies on two steps: expression of a protein carrying the ncAA, and a bioorthogonal reaction between the ncAA and the Fl-dye. We find that protein expression levels do not always correlate with efficient labeling. In some cases, lower expressions of the modified protein resulted in higher SNRs. When labeling cellular structures, such behavior can result from expressing the modified proteins at levels, which are higher than the capacity of the cellular structure. In such cases, the excess of overexpressed protein will remain in the cytosol, leading to increased background levels and consequently lower SNRs. Maintaining low expression levels of the modified protein is preferential for cell physiology. Therefore, upon applying GCE-based labeling, we recommend testing both parameters and choosing the lowest expression conditions that provide the highest SNRs.

The use of the GCE-tag reported here expands the plethora of labeling options for proteins in live cells. As with other labeling approaches, GCE-tag-based bioorthogonal labeling can be combined with conventional labeling approaches for multicolor labeling and

Table 1 List of organelle markers labeled via bioorthogonal labeling using the GCE-tag

\begin{tabular}{llll}
\hline Organelle & Marker name & Fl-dye-Tet & Notes \\
\hline Microtubules & a-Tubulin & SiR & \\
Cell membrane & CAAX & SiR/TAMRA & GFP conjugated \\
Peroxisomes & SKL & SiR/TAMRA & GFP conjugated \\
Lysosomes & Lamp1 & SiR & \\
ER & ER ${ }^{\text {cb5}}$ TM & TAMRA & \\
Exosomes & Exo70 & TAMRA & \\
MVB's & CD63 & TAMRA & \\
\hline
\end{tabular}

can be tailored for super-resolution microscopy [26]. Moreover, the approach can potentially be expanded to labeling endogenous proteins using genome-editing techniques and can be further applied to recently reported GCE-modified tissues and organisms [27-31]. Additionally, a variety of ncAAs that carries different chemical functionalities has been genetically encoded in mammalian cells $[8,32-35]$. The GCE-tag can be further used for incorporating these ncAAs, expanding its use in labeling applications and beyond.

\section{Materials and methods \\ Cell culture}

COS7 and HEK293T cells were kind gifts from Marcelo Ehrlich (Tel Aviv University). Cells were grown in Dulbecco's modified Eagle's medium (DMEM; Life Technologies, Carlsbad, CA) supplemented with $10 \%$ fetal bovine serum (FBS), $2 \mathrm{mM}$ glutamine, 10,000 U/ml penicillin, and $10 \mathrm{mg} / \mathrm{ml}$ streptomycin.

\section{Plasmids and constructs}

Tags were sub-cloned into the single expression vector $\mathrm{pBUD}-\mathrm{BCNK}-\mathrm{RS}$ that carries pylT encoding for tRNA $_{\mathrm{CUA}}{ }^{\text {Pyl }}$, and Pyrrolysyl-tRNA synthetase [18] (Additional file 1: Figure S1), using NotI/KpnI restriction sites. Sequences encoding organelle markers or proteins of interest were then inserted in-frame using the KpnI/XhoI restriction sites at pBUD-BCNK-RS. All constructs were sequenced before use.

\section{Incorporation of the ncAA to proteins in cells}

Assay was performed according to our previously optimized protocol [6]. Twenty-four hours before transfection, cells were plated at $20 \%$ confluency using the following dishes: live cell imaging, 4-well chamber slide (Ibidi, Martinsried, Germany); western blot, 12well plate (NUNC, Rochester, NY); and immunostaining, \#1.0 coverslips (Menzel, Braunschweig, Germany). Cells were transfected with pBUD-BCNK-RS plasmids carrying different tags and organelle markers (Additional file 1: Figure S1, Table S1) using Lipofectamine 2000 (Life Technologies, Carlsbad, CA) according to the manufacturer's protocol, and incubated for $48 \mathrm{~h}$ in the presence of the ncAA BCN-Lys $(0.5 \mathrm{mM}$, Synaffix, Oss, Netherlands) in growth media supplemented with $100 \mu \mathrm{M}$ ascorbic acid (Sigma Aldrich, Israel).

\section{Bioorthogonal labeling}

Forty-eight hours post-transfection, cells were washed with fresh medium $(3 \times$ quick wash followed by $3 \times 30$ min wash) at $37^{\circ} \mathrm{C}$, incubated with SiR-Tet $(1-2 \mu \mathrm{M}, 1$ $h$, Spirochrome, Stein am Rhein, Switzerland) or TAMRA-Tet ( $2 \mu \mathrm{M}, 1 \mathrm{~h}$, Jena BioScience, Germany), and 
washed again with fresh medium $(3 \times$ quick wash and $3 \times 30 \mathrm{~min}$ wash) at $37^{\circ} \mathrm{C}$.

\section{In-gel fluorescence}

Forty-eight hours post-transfection, cells were labeled with the appropriate $\mathrm{Fl}$-dye as described above and in Table 1. After labeling, cells were collected, centrifuged at $200 \times g$ for $5 \mathrm{~min}$, and washed with PBS twice. Then, cells were lysed using RIPA lysis buffer (150 $\mathrm{mM} \mathrm{NaCl}, 1 \%$ NP-40, $0.5 \%$ deoxycholate, $0.1 \%$ SDS, $50 \mathrm{mM}$ Tris [pH 8.0]) supplemented with complete protease inhibitor for $30 \mathrm{~min}$ at $4{ }^{\circ} \mathrm{C}$. Total protein concentrations were measured with BCA Protein Assay Kit (Pierce Biotechnology), and equal total protein amounts were loaded on an SDS-PAGE gel. Gels were imaged at the appropriate wavelength using a Typhoon FLA 7000 biomolecular imager (GE Healthcare, PA, USA) to reveal specific Fl-dye labeling.

\section{Western blot}

Cells were harvested $48 \mathrm{~h}$ post-transfection and lysed using RIPA lysis buffer supplemented with complete protease inhibitor for $30 \mathrm{~min}$ at $4{ }^{\circ} \mathrm{C}$. Total protein concentrations were measured with BCA Protein Assay Kit, and equal total protein amounts were loaded and were subjected to western blot analysis using the following primary antibodies: rabbit anti-HA (1:4000, catalog number G166, Applied Biological Materials, Richmond, Canada, RRID, AB_2813867), mouse anti-GAPDH (1: 1000, catalog number G041, Applied Biological Materials, RRID, AB_2813868), mouse anti-GFP (1:1000, catalog number G096, Applied Biological Materials, RRID, AB_2813869), and rabbit or mouse-peroxidase secondary antibodies $(1: 10,000$, catalog number $715-035-151$ or 711-035-152, Jackson ImmunoResearch, West Grove, PA, RRID, AB_2340771 or AB_10015282).

\section{Immunofluorescence}

Cells were fixed $48 \mathrm{~h}$ post-transfection with $4 \%$ paraformaldehyde (PFA) and co-stained with rabbit anti-HA (1: $500)$ and mouse anti-CD63 (1:200, catalog number ab59479 Abcam, Cambridge, MA, RRID, AB_940915) primary antibodies and with Alexa Fluor 488 anti-rabbit and Alexa Fluor 594 anti-mouse (1:500, catalog number A21206 or A21203 (RRID), Life Technologies; RRID: AB_2535792 or AB_2535789) secondary antibodies. Cells were mounted with Fluoromount-G (SouthernBiotech, Birmingham, AL).

\section{Live cell imaging}

Cells were imaged on a fully incubated confocal spinning-disk microscope at $37^{\circ} \mathrm{C}$ (Marianas; Intelligent Imaging, Denver, $\mathrm{CO}$ ) using a $\times 63$ oil objective (numerical aperture 1.4); depending on the protein expression levels of each cell, the specified Fl-dye or FP was excited at laser powers between 5 and $40 \%$ for $100-350 \mathrm{~ms}$, and recorded on an electron-multiplying charge-coupled device camera (pixel size, $0.079 \mu \mathrm{m}$; Evolve; Photometrics, Tucson, AZ). A total of 3-30 confocal slices were captured for each image.

\section{Image processing}

Analysis of image sets was performed using SlideBook, version 6 (Intelligent Imaging, Denver, CO). To improve visibility, some of the images were subjected to unsharp Mask, Gaussian filter, or both. Care was taken to apply similar filtering for all channels acquired in the same image and for all images that represent the same cellular structure, to allow for comparison. For line intensity analysis, the intensity levels along a line that crosses the cellular structure were measured. Intensity levels were normalized to 1 after background subtraction and plotted. SNRs for each cellular structure were calculated by dividing the maximum intensity by the minimum intensity obtained in each line intensity plot. Intensity levels shown in Fig. $6 \mathrm{~g}$ were calculated by dividing the mean intensity value obtained inside the cell by the mean intensity value measured outside the cell. SNR and intensity measurements for each cellular structure were taken from at least ten different cells from three independent experiments. Plots presented in Fig. 4f, g were generated by segmenting puncta structures using an intensity mask in SlideBook, based on the GFP channel ( $\mathrm{f}$ ) or the SiR channel (g) and plotting the intensity in one channel against the intensity in the other channel. All values were subjected to background subtraction. Pearson's correlation analysis was performed for the indicated regions of interest, using the co-localization plugin in SlideBook. Low intensity levels were filtered out to avoid noise measurements (indicated in green and red lines in plots). Analysis was performed after background subtraction.

\section{FRAP analysis}

FRAP experiments were performed using the same confocal spinning-disk microscope setup, on cells expressing GCE-tag-ER ${ }^{\mathrm{c} 5} \mathrm{TM}$ and labeled with TAMRA-Tet. After four baseline time points, bleaching was carried out on a selected ROI using $100 \mathrm{~ms}$ exposure to a $405 \mathrm{~nm}$ laser. Recovery after bleaching was recorded for $1 \mathrm{~min}$ with 1.8 $\mathrm{s}$ intervals. FRAP analysis fitting and unintentional bleaching corrections were performed using SlideBook.

\section{Statistical analysis}

Statistical analysis was calculated in GraphPad Prism version 5.00 for Windows (La Jolla, CA, USA). Data are shown as means \pm SEM. Statistical significance was determined by ANOVA or $t$ test analysis: ${ }^{* * *} p<0.0001$, "** $p=0.001-0.01, " p=0.01-0.05$, and ns $p>0.05$. 


\section{Supplementary information}

Supplementary information accompanies this paper at https://doi.org/10. 1186/s12915-019-0708-7.

Additional file 1: Figure S1. Single expression vector maps and sequences of the designed linkers. Figure S2. Unsuccessful attempt for MT labeling using Met-TAG-a-tubulin. Figure S3. Unsuccessful attempt of labeling of MVBs, Exosomes and ER using the GCE-tag and SiR-Tet labeling. Figure S4. Unsuccessful mitochondria labeling using the GCE-tag. Figure S5. Labeling cellular proteins using the GCE-tag. Figure S6. Evaluating peroxisomes, exosomes and MTs labeling using Flag/Myc- GGSG-TAG or GGSG-TAG as

GCE-tags. Table S1. Sequences of organelle markers used in this work.

Additional file 2: Movie S1. Microtubule dynamics in cells labeled with GCE-tag-a-tubulin. COS7 cells expressing GCE-tag-a-tubulin and labeled with SiR-Tet were recorded at $4 \mathrm{~s}$ intervals. Shown are maximum intensity projections of 3 z-slices taken from a representative cell. Scale-bar: $10 \mu \mathrm{m}$.

Additional file 3: Movie S2. Plasma membrane dynamics in cells labeled with GCE-tag-GFP-CAAX. COS7 cells expressing GCE-tag-GFPCAAX were labeled with SiR-Tet and incubated in serum-free DMEM for $6 \mathrm{~h}$. Cells were recorded at $5.5 \mathrm{~s}$ intervals. Left panel: 640 (SiR, red) channel, middle panel: 488 (GFP, green) channel, right panel: overly. Shown are maximum intensity projections of 3 z-slices taken from a representative cell. Scale-bar: $10 \mu \mathrm{m}$

Additional file 4: Movie S3. Peroxisome dynamics in cells labeled with GCE-tag-GFP-SKL. COS7 cells expressing GCE-tag-GFP-SKL and labeled with SiR-Tet were recorded at $5.3 \mathrm{~s}$ intervals. Left panel: 488 (GFP, green) channel, middle panel: 640 (SiR, red) channel. Shown are maximum inten sity projections of $30 \mathrm{z}$-slices taken from a representative cell. Scale-bar: $10 \mu \mathrm{m}$.

Additional file 5: Movie S4. Lysosome dynamics in cells labeled with GCE-tag-Lamp1 and treated with chloroquine. COS7 cells expressing GCE-tag-Lamp1 and labeled with SiR-Tet were imaged for $3 \mathrm{~h}$ in the presence of chloroquine $(120 \mu \mathrm{M})$, at 10 min intervals. Shown are maximum intensity projections of $20 \mathrm{z}$-slices taken from a representative cell. Scalebar: $10 \mu \mathrm{m}$.

Additional file 6: Movie S5. MVB dynamics in cells labeled with GCEtag-CD63. COS7 cells expressing GCE-tag-CD63 and labeled with TAMRATet were recorded at $0.4 \mathrm{~s}$ intervals. Shown are maximum intensity projections of $20 \mathrm{z}$-slices taken from a representative cell. Scale-bar: $10 \mu \mathrm{m}$.

Additional file 7: Movie S6. Exosome dynamics in cells expressing GCE-tag-Exo70. COS7 cells expressing GCE-tag-Exo70 and labeled with TAMRA-Tet were recorded at $1 \mathrm{~s}$ intervals. Single confocal slices taken from a representative movie are shown. Scale-bar: $10 \mu \mathrm{m}$.

Additional file 8: Movie S7. Visualizing ER dynamics by applying FRAP to GCE-tag-ER ${ }^{\mathrm{cb} 5}$ TM labeled ER. COS7 cells expressing GCE-tag-ER ${ }^{\mathrm{cb} 5} \mathrm{TM}$ labeled with TAMTA-Tet were imaged for 2 min with $2 \mathrm{~s}$ intervals. Photobleaching was performed after 4 baseline timepoints ( $12 \mathrm{~s}$ ). Shown are maximum intensity projections of $3 z$-slices taken from a representative cell. Scale-bar: $10 \mu \mathrm{m}$.

Additional file 9: Movie S8. A Zoomed-in video of the bleached region in the ER. A Zoomed-in video of the bleached region shown in Additional file 8: Movie S7. Scale-bar: $2 \mu \mathrm{m}$.

\section{Acknowledgements}

We thank Peter Kim (University of Toronto) and Jennifer Lippincott Schwartz (Janelia Research campus) for contributing plasmids for fluorescent proteinbased organelle markers. We also thank all members of the Elia lab for critical reading of the manuscript.

\section{Authors' contributions}

IS and NE designed and analyzed all the experiments. IS performed most experiments. DN performed the biochemical experiments and provided technical support. AK and AA performed the experiments. EA advised on the GCE. NE, EA, and DN initiated the project. NE wrote the manuscript. All authors read and approved the final manuscript.

\section{Funding}

The project leading to this application has received funding from the European Research Council (ERC) under the European Union's Horizon 2020 research.

\section{Availability of data and materials}

All data generated or analyzed during this study are included in this published article and its supplementary information files.

Ethics approval and consent to participate

No ethics approval was required for this study.

\section{Competing interests}

The authors declare that they have no competing interests.

\section{Author details}

${ }^{1}$ Department of Life Sciences, Ben-Gurion University of the Negev, 84105 Beer Sheva, Israel. ' National Institute for Biotechnology in the Negev (NIBN), Ben-Gurion University of the Negev, 84105 Beer Sheva, Israel. ${ }^{3}$ Department of Chemistry, Ben-Gurion University of the Negev, 84105 Beer Sheva, Israel.

Received: 29 June 2019 Accepted: 15 October 2019

Published online: 14 January 2020

\section{References}

1. $\quad$ van de Linde S, Heilemann M, Sauer M. Live-cell super-resolution imaging with synthetic fluorophores. Annu Rev Phys Chem. 2012;63:519-40.

2. Lang K, Chin JW. Cellular incorporation of unnatural amino acids and bioorthogonal labeling of proteins. Chem Rev. 2014;114(9):4764-806.

3. Nikic I, Kang JH, Girona GE, Aramburu IV, Lemke EA. Labeling proteins on live mammalian cells using click chemistry. Nat Protoc. 2015;10(5): 780-91

4. Uttamapinant C, Howe JD, Lang K, Beranek V, Davis L, Mahesh M, Barry NP, Chin JW. Genetic code expansion enables live-cell and super-resolution imaging of site-specifically labeled cellular proteins. J Am Chem Soc. 2015;137(14):4602-5

5. Sakin V, Hanne J, Dunder J, Anders-Osswein M, Laketa V, Nikic I, Krausslich HG, Lemke EA, Muller B. A versatile tool for live-cell imaging and superresolution nanoscopy studies of HIV-1 Env distribution and mobility. Cell Chem Biol. 2017;24(5):635-45 e635.

6. Schvartz T, Aloush N, Goliand I, Segal I, Nachmias D, Arbely E, Elia N. Direct fluorescent-dye labeling of alpha-tubulin in mammalian cells for live cell and superresolution imaging. Mol Biol Cell. 2017;28(21):2747-56.

7. Neubert F, Beliu G, Terpitz U, Werner C, Geis C, Sauer M, Doose S. Bioorthogonal click chemistry enables site-specific fluorescence labeling of functional NMDA receptors for super-resolution imaging. Angew Chem. 2018;57(50):16364-9.

8. Peng T, Hang HC. Site-specific bioorthogonal labeling for fluorescence imaging of intracellular proteins in living cells. J Am Chem Soc. 2016; 138(43):14423-33.

9. Lang K, Davis L, Wallace S, Mahesh M, Cox DJ, Blackman ML, Fox JM, Chin JW. Genetic encoding of bicyclononynes and trans-cyclooctenes for sitespecific protein labeling in vitro and in live mammalian cells via rapid fluorogenic Diels-Alder reactions. J Am Chem Soc. 2012;134(25):10317-20.

10. Vreja IC, Nikic I, Gottfert F, Bates M, Krohnert K, Outeiro TF, Hell SW, Lemke EA, Rizzoli SO. Super-resolution microscopy of clickable amino acids reveals the effects of fluorescent protein tagging on protein assemblies. ACS Nano. 2015;9(11):11034-41

11. Davis L, Chin JW. Designer proteins: applications of genetic code expansion in cell biology. Nat Rev Mol Cell Biol. 2012;13(3):168-82.

12. Johnson JA, Lu YY, Van Deventer JA, Tirrell DA. Residue-specific incorporation of non-canonical amino acids into proteins: recent developments and applications. Curr Opin Chem Biol. 2010;14(6):774-80.

13. Lang K, Davis L, Torres-Kolbus J, Chou C, Deiters A, Chin JW. Genetically encoded norbornene directs site-specific cellular protein labelling via a rapid bioorthogonal reaction. Nat Chem. 2012:4(4):298-304.

14. Baskin JM, Prescher JA, Laughlin ST, Agard NJ, Chang PV, Miller IA, Lo A, Codelli JA, Bertozzi CR. Copper-free click chemistry for dynamic in vivo imaging. Proc Natl Acad Sci U S A. 2007;104(43):16793-7.

15. Hostetler ZM, Ferrie Jل Jornstein MR, Sungwienwong IL Petersson EJ, Kohli RM. Systematic evaluation of soluble protein expression using a fluorescent 
unnatural amino acid reveals no reliable predictors of tolerability. ACS Chem Biol. 2018;13(10):2855-61.

16. Nikic I, Estrada Girona G, Kang JH, Paci G, Mikhaleva S, Koehler C, Shymanska NV, Ventura Santos C, Spitz D, Lemke EA. Debugging eukaryotic genetic code expansion for site-specific click-PAINT super-resolution microscopy. Angew Chem. 2016;55(52):16172-6.

17. Chin JW. Expanding and reprogramming the genetic code. Nature. 2017;550(7674):53-60.

18. Cohen S, Arbely E. Single-plasmid-based system for efficient noncanonical amino acid mutagenesis in cultured mammalian cells. Chembiochem. 2016; 17(11):1008-11.

19. Aloush N, Schvartz T, Konig Al, Cohen S, Brozgol E, Tam B, Nachmias D, BenDavid O, Garini Y, Elia N, et al. Live cell imaging of bioorthogonally labelled proteins generated with a single pyrrolysine tRNA gene. Sci Rep. 2018;8(1): 14527.

20. Lukinavicius G, Umezawa K, Olivier N, Honigmann A, Yang G, Plass T, Mueller V, Reymond L, Correa IR Jr, Luo ZG, et al. A near-infrared fluorophore for live-cell super-resolution microscopy of cellular proteins. Nat Chem. 2013;5(2):132-9.

21. Kolodziej PA, Young RA. Epitope tagging and protein surveillance. Methods Enzymol. 1991;194:508-19.

22. Yoon YH, Cho KS, Hwang JJ, Lee SJ, Choi JA, Koh JY. Induction of lysosomal dilatation, arrested autophagy, and cell death by chloroquine in cultured ARPE-19 cells. Invest Ophthalmol Vis Sci. 2010;51(11):6030-7.

23. Fu C, Kobayashi T, Wang N, Hoppmann C, Yang B, Irannejad R, Wang L. Genetically encoding quinoline reverses chromophore charge and enables fluorescent protein brightening in acidic vesicles. J Am Chem Soc. 2018; 140(35):11058-66.

24. Snapp EL, Altan N, Lippincott-Schwartz J. Measuring protein mobility by photobleaching GFP chimeras in living cells. Curr Prot Cell Biol. 2003; Chapter 21:Unit 2121.

25. Martin BR, Giepmans BN, Adams SR, Tsien RY. Mammalian cell-based optimization of the biarsenical-binding tetracysteine motif for improved fluorescence and affinity. Nat Biotechnol. 2005;23(10):1308-14.

26. Beliu G, Kurz AJ, Kuhlemann AC, Behringer-Pliess L, Meub M, Wolf N, Seibe J, Shi ZD, Schnermann M, Grimm JB, et al. Bioorthogonal labeling with tetrazine-dyes for super-resolution microscopy. Commun Biol. 2019;2:261.

27. Greiss S, Chin JW. Expanding the genetic code of an animal. J Am Chem Soc. 2011:133(36):14196-9.

28. Bianco A, Townsley FM, Greiss S, Lang K, Chin JW. Expanding the genetic code of Drosophila melanogaster. Nat Chem Biol. 2012;8(9):748-50.

29. Ernst RJ, Krogager TP, Maywood ES, Zanchi R, Beranek V, Elliott TS, Barry NP, Hastings MH, Chin JW. Genetic code expansion in the mouse brain. Nat Chem Biol. 2016;12(10):776-8

30. Brown W, Liu J, Tsang M, Deiters A. Cell-lineage tracing in zebrafish embryos with an expanded genetic code. Chembiochem. 2018;19(12):1244-9.

31. Brown W, Liu J, Deiters A. Genetic code expansion in animals. ACS Chem Biol. 2018:13(9):2375-86.

32. Koehler C, Sauter PF, Wawryszyn M, Girona GE, Gupta K, Landry JJ, Fritz MH, Radic K, Hoffmann JE, Chen ZA, et al. Genetic code expansion for multiprotein complex engineering. Nat Methods. 2016;13(12):997-1000.

33. Italia JS, Zheng Y, Kelemen RE, Erickson SB, Addy PS, Chatterjee A. Expanding the genetic code of mammalian cells. Biochem Soc Trans. 2017; 45(2):555-62.

34. Brabham R, Fascione MA. Pyrrolysine amber stop-codon suppression: development and applications. Chembiochem. 2017;18(20):1973-83.

35. Dumas A, Lercher L, Spicer CD, Davis BG. Designing logical codon reassignment - expanding the chemistry in biology. Chem Sci. 2015;6(1):50-69.

\section{Publisher's Note}

Springer Nature remains neutral with regard to jurisdictional claims in published maps and institutional affiliations.

Ready to submit your research? Choose BMC and benefit from:

- fast, convenient online submission

- thorough peer review by experienced researchers in your field

- rapid publication on acceptance

- support for research data, including large and complex data types

- gold Open Access which fosters wider collaboration and increased citations

- maximum visibility for your research: over $100 \mathrm{M}$ website views per year

At $\mathrm{BMC}$, research is always in progress.

Learn more biomedcentral.com/submissions 